\title{
Invariant Theory and Differential Equations
}

\author{
Peter J. Olver† \\ School of Mathematics \\ University of Minnesota \\ Minneapolis, $\mathrm{MN}$ \\ USA $\quad 55455$
}

In: Invariant Theory, S.S. Koh, ed., Lecture Notes in Math, vol. 1278, Springer-Verlag, New York, 1987, pp. 62-80.

$†$ Research Supported in Part by NSF Grant DMS 86-02004. 


\section{Introduction}

Recent years have witnessed a resurgence of interest in classical invariant theory. In the field of differential equations, it has become of steadily increasing importance, not only in the applications to be discussed here, but also in the theory of canonical forms of Hamiltonian systems, [4], and the study of conservation laws, [20]. The present work originally arose in the study of nonconvex variational problems of interest in elasticity for which one could prove existence of weak minimizers. A transform introduced by Gel'fand and Dikii, [7], and Shakiban, [19], changes this problem into one about the primality of certain determinantal ideals, and thus provides a complete solution. Subsequently the transform method has been applied to a wide range of problems arising in the study of differential equations and the calculus of variations. It has also been recognized, [14], that when the functions are homogeneous polynomials, the transform method is equivalent to the classical symbolic method of invariant theory. This paper will review the transform method, its relationship and application to classical invariant theory, and its application to problems arising in the calculus of variations. The last section provides a brief summary of a new, and potentially important theory of higher order differential forms ("hyperforms") which has arisen in an attempt to understand new divergence identities for transvectants which are a direct result of these investigations.

Throughout, we will let $\mathrm{x}=\left(\mathrm{x}^{1}, \ldots, \mathrm{x}^{\mathrm{p}}\right)$ be the independent variables and $\mathrm{u}=$ $\left(u^{1}, \ldots, u^{q}\right)$ be the dependent variables in some system of differential equations, so that the u's are to be viewed as functions of the x's. Partial derivatives will be denoted by subscripts, e.g. $u_{12}=\partial^{2} u / \partial x^{1} \partial x^{2}$, or more generally using multi-index notation, so $u_{I}^{\alpha}$ for $I=\left(i_{1}, \ldots, i_{k}\right)$ will denote the $k$-th order partial derivative $\partial^{k}{ }^{\alpha} / \partial x^{i}{ }_{1} \ldots \partial x^{i} k$. A differential polynomial is a complex-valued polynomial in the derivatives $u_{I}^{\alpha}$ (for simplicity we are excluding explicit $\mathrm{x}$ dependence in our differential polynomials). A differential polynomial is called differentially homogeneous of order $\mathrm{k}$ if it depends exclusively on derivatives of order $\mathrm{k}$; algebraically homogeneous refers to the usual concept of homogeneity for polynomials. Thus $\mathrm{u}_{11} \mathrm{u}_{22} \mathrm{u}_{12}$ is differentially homogeneous of degree 2 , but not algebraically homogeneous, whereas $u_{1} u_{22}-u_{2} u_{11}$ is algebraically homogeneous of degree 2 , but not differentially homogeneous. We let $D_{i} P$ denote the total derivative of the differential polynomial $P$ with respect to $x^{i}$, meaning that we differentiate $P$ treating the $u$ 's as functions of the $x$ 's. For instance, $D_{2}\left(u_{1} u_{23}\right)=$ 
$u_{12} u_{23}+u_{1} u_{223}$. The total divergence of a $p$-tuple $P=\left(P_{1}, \ldots, P_{p}\right)$ of differential polynomials is the differential polynomial

$$
\text { Div } P=D_{1} P_{1}+\ldots+D_{p} P_{p}
$$

The following questions are of importance for applications:

1) Characterize all differentially homogeneous differential polynomials $Q$ which can be written as divergences: $Q=$ Div $P$ for some $p$-tuple $P$. An important example is the Jacobian determinant

$$
\frac{\partial(u, v)}{\partial\left(x^{1}, x^{2}\right)}=u_{1} v_{2}-u_{2} v_{1}=D_{1}\left(u v_{2}\right)+D_{2}\left(-u v_{1}\right)
$$

2) Characterize all differentially homogeneous null divergences, meaning $p$-tuples of differential polynomials satisfying the identity Div $P=0$ for all functions $u(x)$. An example is the "Jacobian identity"

$$
D_{1}\left(u_{2} v_{3}-u_{3} v_{2}\right)+D_{2}\left(u_{3} v_{1}-u_{1} v_{3}\right)+D_{3}\left(u_{1} v_{2}-u_{2} v_{1}\right)=0
$$

3) More generally, characterize all non-negative divergences, meaning $\mathrm{p}$-tuples of differential polynomials satisfying the identity $\operatorname{Div} P \geq 0$ for all $u$.

4) Characterize all differentially homogeneous differential polynomials $Q$ which can be written as higher order divergences: $Q=$ Div $^{k} P$. More explicitly, we want to write

$$
Q=\sum D_{I} P_{I}
$$

for certain differential polynomials $P_{\mathrm{I}}$. Here the sum is over all $\mathrm{k}^{\text {th }}$ order multi-indices $\mathrm{I}=\left(\mathrm{i}_{1}, \ldots, \mathrm{i}_{\mathrm{k}}\right), 1 \leq \mathrm{i}_{v} \leq \mathrm{p}$, with $\mathrm{D}_{\mathrm{I}}$ denoting the corresponding $\mathrm{k}^{\text {th }}$ order total derivative $D_{i_{1}} \ldots D_{i_{k}}$. An important example is the Hessian of a function $u$, which is a second order divergence:

$$
u_{11} u_{22}-u_{12}^{2}=D_{1}^{2}\left(-u_{2}^{2}\right)+D_{1} D_{2}\left(u_{1} u_{2}\right)+D_{2}^{2}\left(-u_{1}^{2}\right)
$$

Problem 1 arises in Ball's theory of polyconvex variational problems, which are of great interest in elasticity; the solution is essentially that all such homogeneous divergences are linear combinations of Jacobian deteminants, [2]. Problem 2 arises in the classification of conservation laws of partial differential equations, where the null divergences are known 
as trivial conservation laws or, ocassionally, strong conservation laws since they hold for all functions $u$; the solution is that all such p-tuples are linear combinations of certain natural generalizations of the basic Jacobian identity given above, [16]. Problem 3 arises in the theory of continuum thermomechanics, where the Coleman-Noll procedure, [3], applied to the basic inequality arising from the second law of thermodynamics, results in such divergence inequalities; here the solution is essentially that any non-negative divergence must actually be a null divergence, and so problem 3 reduces to problem 2, [17]. This result was recently used by Dunn and Serrin, [6], in their theory of interstitial working. Finally, problem 4, which is the most interesting from the point of view of classical invariant theory, arose in generalizations of the applications of problem 1 to the variational problems of elasticity, and was used to produce nonconvex variational problems with rather weak coercivity conditions for which it was still possible to prove the existence of weak minimizers, [14]. The solution to this last problem, to be explained in more detail below, is that such a differential polynomial must be a linear combination of $\mathbf{k}^{\text {th }}$ order transvectants of the functions $\mathrm{u}$ and their derivatives, the Hessian being a multiple of the second order transvectant $(u, u)^{(2)}$, in the case that $u$ is a homogeneous polynomial function.

\section{The Transform.}

The key to the solution of the above problems is the introduction of a transform which, like the Fourier transform of classical analysis, changes questions about derivatives and differential polynomials into questions about ordinary algebraic polynomials, thus making them amenable to the powerful techniques of commutative algebra and invariant theory. A special case of this transform was introduced by Gel'fand and Dikii, [7], in connection with the Korteweg-deVries equation and the formal calculus of variations. It was generalized by Shakiban, [19], [20], and used to apply the invariant theory of finite groups to the study of conservation laws of differential equations. The present version is essentially the same as that discussed by Ball, Currie and Olver, [2], in the solution of the first and fourth problems of section 1. Subsequently, [14], this transform was, in the

special case of polynomial functions $u$, recognized to be equivalent to the standard symbolic method of classical invariant theory.

In order to introduce the transform, it is easiest to start with the case when there is just one dependent variable $u$ (so $q=1$ ), depending on $p$ independent variables. Consider an algebraically homogeneous differential polynomial $\mathrm{P}$ of degree $\mathrm{r}$ (but not 
necessarily differentially homogeneous). Its transform, $\hat{P}=\mathcal{F}(\mathrm{P})$, will be an algebraic polynomial $\hat{P}(Z)$ of the $r \times p$ matrix of independent variables $Z=\left(z_{j}^{i}\right), 1 \leq i \leq p, 1 \leq j \leq$ r. The explicit formula for $\hat{P}$ is determined as follows: If $\mathbf{P}$ is a linear differential polynomial, so $\mathrm{r}=1$, then $\mathrm{Z}=\left(\mathrm{z}^{1}, \ldots, \mathrm{z}^{\mathrm{p}}\right)$ is a single row vector, and $\hat{P}(\mathrm{Z})$ is the ordinary Fourier transform $\hat{P}(k)$ of $P$ when $z^{j}=\sqrt{-1} k^{j}$. For example, if $P=u_{11}+u_{22}$, then $\mathcal{F}(P)=\left(z^{1}\right)^{2}+\left(z^{2}\right)^{2}$. In general, $\mathcal{F}\left(u_{I}\right)=z^{I}$, where, for $I=\left(i_{1}, \ldots, i_{k}\right), z^{I}=$ $z^{i} 1 \cdot z^{i} \cdot \ldots \cdot z^{i} k$. For higher degree differential polynomials, a natural first try for $\mathcal{F}$ would be to Fourier transform each derivative of $u$ using a different row of the matrix $Z$ to distinguish them; in other words, try $\mathcal{F}\left(u_{I_{1}} \cdot \ldots \cdot u_{I_{r}}\right)=z_{1}^{I_{1}} \cdot \ldots \cdot z_{r} I_{r}$. However, this is ambiguous, since we can commute the $u_{I_{m}}$ 's. This ambiguity can be easily resolved by introducing the symmetrizing map $\sigma=\frac{1}{\mathrm{r} !} \sum \pi$, where the sum is over all permutations $\pi$ of the integers $\{1, \ldots, \mathrm{r}\}$, and where $\pi(\mathrm{Z})=\left(\mathrm{z}_{\mathrm{i}}^{\pi(\mathrm{j})}\right), \pi[\hat{\mathrm{P}}(\mathrm{Z})]=\hat{\mathrm{P}}(\pi(\mathrm{Z}))$. Thus, define the transform of an $\mathrm{r}^{\text {th }}$ degree differential monomial to be

$$
\mathcal{F}\left(\mathrm{u}_{\mathrm{I}_{1}} \cdot \ldots \cdot \mathrm{u}_{\mathrm{I}_{\mathrm{r}}}\right)=\sigma\left\{\mathrm{z}_{1}^{\mathrm{I}_{1}} \cdot \ldots \cdot \mathrm{z}_{\mathrm{r}}^{\mathrm{I}}\right\}
$$

and extend $\mathcal{F}$ by linearity.

Example. For the Hessian, $P=u_{11} u_{22}-u_{12}^{2}$, we have $p=r=2$, so $Z$ is a $2 \times 2$ matrix of variables, which for simplicity we denote by

$$
\mathrm{Z}=\left(\begin{array}{cc}
\mathrm{z}^{1} & \mathrm{z}^{2} \\
\mathrm{w}^{1} & \mathrm{w}^{2}
\end{array}\right)
$$

We find that

$$
\begin{aligned}
\mathcal{F}(P)= & \sigma\left\{\left(z^{1}\right)^{2}\left(w^{2}\right)^{2}-z^{1} z^{2} w^{1} w^{2}\right\} \\
& =\frac{1}{2}\left\{\left(z^{1}\right)^{2}\left(w^{2}\right)^{2}+\left(z^{2}\right)^{2}\left(w^{1}\right)^{2}-2 z^{1} z^{2} w^{1} w^{2}\right\} \\
& =\frac{1}{2}(\operatorname{det} Z)^{2} .
\end{aligned}
$$

Theorem. The transform $\mathcal{F}$ determines a linear isomorphism from the space of algebraically homogeneous differential polynomials to the space of symmetric algebraic 
polynomials of the matrix of variables $\mathrm{Z}$. (By definition, $\hat{\mathrm{P}}(\mathrm{Z})$ is symmetric if $\pi(\hat{\mathrm{P}})=\hat{\mathrm{P}}$ for all permutations $\pi$.)

More generally, if there is more than one dependent variable, so $q>1$, then we define the transform on differential polynomials which are homogeneous of degree $r_{\alpha}$ in $u^{\alpha}$, where $r=r_{1}+\ldots+r_{q}$, simply by writing the $u^{\prime} s$ in each differential monomial in ascending order, and, instead of using the full symmetrizing map $\sigma$, just using $\tilde{\sigma}=$ $\left(\Pi_{v} !\right)^{-1} \Sigma^{\prime} \pi$, the sum now being only over those permutations which permute each set of $r_{\alpha}$ rows of $Z$ among themselves. For instance, if $P$ depends quadratically on derivatives of $u^{1}$ and linearly on derivatives of $u^{2}$, then $r=3$, and the only two permutations occuring in $\tilde{\sigma}$ are the identity and (12). The isomorphism theorem, with the proper interpretation of "symmetric", works just as before. (This is equivalent to, but slightly different from, the procedure used in [2], [14].)

The key to the utility of the transform method is its ability to change differential operations into algebraic operations. Two particularly important operations are the total derivatives $D_{i}$, and the Euler operator (variational derivative) $E$ from the calculus of variations, cf. [7]. If $\mathrm{L}$ is any linear operator on the space of differential polynomials, then we let $\hat{L}$ be the corresponding "transformed" operator on the space of symmetric polynomials $\hat{\mathrm{P}}(\mathrm{Z})$.

Proposition. The transforms of the total derivatives $D_{i}$ and the Euler operator $E$ are given by

$$
\hat{D}_{i} \hat{P}=\left(z_{1}^{i}+\ldots+z_{r}^{i}\right) \cdot \hat{P}(Z),
$$

and, letting $z_{j}$ denote the $j^{\text {th }}$ row of $Z$,

$$
\hat{E}(\hat{P})=\hat{P}\left(z_{1}, \ldots, z_{r-1},-z_{1}-\ldots-z_{r-1}\right) .
$$

(Note that $\mathrm{E}(\mathrm{P})$ has algebraic degree one less than that of $\mathrm{P}$.)

As an immediate corollary, we derive the well-known result, cf. [7], that the kernel of the Euler operator is the image of the total divergence:

$$
E(P)=0 \quad \text { if and only if } \quad P=\operatorname{Div} Q \text { for some } p \text {-tuple } Q \text {. }
$$


Indeed, transforming the above statement, we see that it is equivalent to the trivial algebraic proposition

$\hat{\mathrm{P}}\left(\mathrm{z}_{1}, \ldots, \mathrm{z}_{\mathrm{r}-1},-\mathrm{z}_{1}-\ldots-\mathrm{z}_{\mathrm{r}-1}\right)=0 \quad$ if and only if $\quad \hat{\mathrm{P}}(\mathrm{Z})=\sum_{\mathrm{i}}\left(\mathrm{z}_{\mathrm{i}}^{1}+\ldots+\mathrm{z}_{\mathrm{i}}^{\mathrm{r}}\right) \cdot \hat{\mathrm{Q}}_{\mathbf{i}}$

for polynomials $\hat{Q}_{\mathrm{i}}(\mathrm{Z})$.

\section{The Symbolic Method.}

In the special case when each function $u^{\alpha}=\sum\left(\begin{array}{l}m \\ I\end{array}\right) \cdot c_{I}^{\alpha} \cdot x^{I}$ is a homogeneous polynomial or form of degree $\mathrm{m}$, a differential polynomial $\mathrm{P}[\mathrm{u}]$ will evaluate to a polynomial function of the coefficients $c_{I}^{\alpha}$ as well as the $x$ 's. As such, it will have an umbral representation determined by the classical symbolic method, cf. [8], [9], [10]. Here we will follow Gurevich's notation for symbolic factors of the first and second kind, as the convention of Kung and Rota in which they are both brackets is rather special to the case of binary forms $(p=2)$. Thus, if $z_{j}=\left(z_{j}^{1}+\ldots+z_{j}^{p}\right), j=1, \ldots, r$, are umbral letters (the reason we use $\mathrm{z}$ rather than the more standard Greek letters will be clear presently), we use the notation

$$
\left(z_{j} x\right)=z_{j}^{1} x^{1}+\ldots+z_{j}^{p} x^{p}
$$

for the symbolic factors of the first kind, and

$$
\left[z_{j_{1}}, \ldots, z_{j_{p}}\right]=\operatorname{det}\left(z_{j_{v}}^{i}\right) \equiv \operatorname{det}\left(Z_{J}\right)
$$

for the symbolic factors of the second kind, or bracket factors. Note that if we form an $\mathrm{r} \times \mathrm{p}$ matrix $\mathrm{Z}$ out of the letters $\mathrm{z}_{1}, \ldots, \mathrm{z}_{\mathrm{r}}$, then the bracket factor $\left[\mathrm{z}_{\mathrm{j}_{1}}, \ldots, \mathrm{z}_{\mathrm{j}_{\mathrm{p}}}\right]$ can be identified with the determinant of the $p \times p$ minor $Z_{J}$ consisting of rows $j_{1}, \ldots, j_{p}$ of $Z$.

A key observation is that, apart from inessential symbolic factors of the first kind and a multiplicative constant, the transform of $P$ agrees with the unique symmetric umbral representation of $P$. Specifically, we have the following result.

Theorem. Let $\mathrm{P}[\mathrm{u}]$ be a differential polynomial, which is both differentially homogeneous of degree $k$ and algebraically homogeneous of degree $r_{\alpha}$ in the $k^{\text {th }}$ derivatives of $u^{\alpha}$, with $r=r_{1}+\ldots+r_{q}$. Let $\hat{P}(Z)$ be the transform of $P$. Let $u^{\alpha}(x)$ be 
(different) homogeneous $\mathrm{m}^{\text {th }}$ order polynomial functions, and let $\mathrm{P}[\mathrm{u}(\mathrm{x})]$ denote the evaluation of $P$ when $u^{\alpha}=u^{\alpha}(x)$. Then the function

$$
\hat{P}(Z) \cdot\left\{\frac{m !}{(m-k) !}\right\} \cdot \prod_{j=1}^{r}\left(z_{j} x\right)^{m-k}
$$

is the symmetric umbral representation of $P[u(x)]$, in which we are viewing the first $r_{1}$ rows of $Z$ as sets of equivalent letters corresponding to $u^{1}$, the second $r_{2}$ rows as equivalent letters corresponding to $\mathrm{u}^{2}$, etc., and $\left(z_{j} x\right)$ is the symbolic factor of the first kind corresponding to the letter $z_{j}$ which forms the $j^{\text {th }}$ row of $Z$.

Conversely, if $\hat{Q}(Z)$ is a symmetric umbral representative of a polynomial $Q(x)$ depending on a collection of forms $u^{1}, \ldots, u^{q}$, and the only appearance of $x$ in $\hat{Q}$ is through $(m-k)^{\text {th }}$ powers of symbolic factors of the first kind, then there is an equivalent transform polynomial $\hat{P}(Z)$ related to $\hat{Q}$ by $(*)$ such that if $P[u]$ is the corresponding differential polynomial, then $P$ has evaluation $P[u(x)]=Q(x)$.

This readily generalizes to the case when $P$ is not differentially homogeneous, or when the forms $u^{\alpha}$ have different degrees, in which case the symbolic factors of the first kind occur with different powers; we leave the general statement to the interested reader.

This theorem gives an effective and simple procedure for answering problem 7 in Kung and Rota's paper, [10], on how to find the differential polynomial corresponding to such a symbolic expression. This is done by: first, ignoring the inessential symbolic factors of the first kind, second, dividing through by the appropriate product of factorials, and, third, undoing the transform $\mathcal{F}$. This last step is also quite elementary to accomplish. (There are, of course, other symbolic expressions depending on $x$ and the coefficients of the forms which cannot not be written solely in terms of symbolic factors of the first kind, but a) they are never invariants, and b) they cannot be written as constant coefficient differential polynomials)

Example. The Hessian of a binary form degree m, i.e. $u_{11} u_{22}-u_{12}^{2}$, has the umbral representation

$$
\hat{H}=\frac{1}{2} m^{2}(m-1)^{2}[z w]^{2}(z x)^{m-2}(w x)^{m-2},
$$


cf. [10; page 70]. Comparing with $\left(^{*}\right)$, we see that the corresponding transform polynomial is just

$$
\hat{P}=\frac{1}{2}[z w]^{2}=\frac{1}{2}(\operatorname{det} Z)^{2},
$$

where $\mathrm{Z}$ is the above $2 \times 2$ matrix. Thus we recover the standard representation of the Hessian of a form as a polynomial in its second order derivatives.

As a second example, consider the transvectant $(u, u)^{(4)}$, which has umbral representation

$$
\hat{\mathrm{T}}=\frac{1}{2}[\mathrm{z} \mathrm{w}]^{4}(\mathrm{zx})^{\mathrm{m}-4}(\mathrm{w} \mathrm{x})^{\mathrm{m}-4}
$$

The corresponding transform polynomial is, according to $(*)$,

$$
\begin{gathered}
\hat{P}=\frac{(m-4) !^{2}}{2 \cdot m !^{2}}[z w]^{4}=\frac{(m-4) !^{2}}{2 \cdot m !^{2}}(\operatorname{det} Z)^{4} \\
=\frac{(m-4) !^{2}}{2 \cdot m !^{2}}\left\{\left(z^{1}\right)^{4}\left(w^{2}\right)^{4}-6\left(z^{1}\right)^{3} z^{2} w^{1}\left(w^{2}\right)^{3}+10\left(z^{1}\right)^{2}\left(z^{2}\right)^{2}\left(w^{1}\right)^{2}\left(w^{2}\right)^{2}-\right. \\
\left.-6 z^{1}\left(z^{2}\right)^{3}\left(w^{1}\right)^{3} w^{2}+\left(z^{2}\right)^{4}\left(w^{1}\right)^{4}\right\},
\end{gathered}
$$

It is easy to see that this is the transform of the differential polynomial

$$
P=\frac{(m-4) !^{2}}{m !^{2}}\left\{u_{1111} u_{2222}-6 u_{1112} u_{1222}+5 u_{1122}^{2}\right\}
$$

If we had done the transvectant $(u, v)^{(4)}$ of two different forms of degree $\mathrm{m}$, then we would recover the classical formula

$$
\frac{(m-4) !^{2}}{m !^{2}}\left\{u_{1111} v_{2222}-6 u_{1112} v_{1222}+10 u_{1122} v_{1122}-6 u_{1222} v_{1112}+u_{2222} v_{1111}\right\}
$$

cf. [8; page 227]. This clearly demonstrates the power of the transform method for determining the differential polynomial expressions of formulas from classical invariant theory. 
In fact, the transform approach to classical invariant theory accomplishes more. Since it works for arbitrary smooth $\left(C^{\infty}\right)$ functions, not just homogeneous polynomials, it leads immediately to an invariant theory of smooth (or analytic) functions. In this case, the invariants to be considered are differential polynomials in the functions, which are unchanged (up to a factor) under the action of the general linear group GL(p). It is not difficult to see that the induced action of GL(p) on the space of differential polynomials commutes with the transform. Consequently, the First Fundamental Theorem of Classical Invariant Theory, [9], [10], immediately provides a classification of all invariant differential polynomials.

Theorem. A differential polynomial $\mathrm{P}[\mathrm{u}]$ is an invariant under the general linear group GL(p) if and only if its transform $\hat{P}(Z)$ is a polynomial in the symbolic factors of the second kind, i.e. a bracket polynomial. In particular, this requires that $r$, the degree of algebraic homogeneity of $P$, be greater than or equal to $p$, the number of independent variables, and that $\hat{\mathrm{P}}$ be a polynomial in the determinants of the $\mathrm{p} \times \mathrm{p}$ minors of the $\mathrm{r} \times \mathrm{p}$ matrix Z.

There are, of course, relations among the invariant differential polynomials, all stemming from the basic syzygy among the brackets:

$$
\sum_{j=1}^{p+1}\left[z_{1}, \ldots, z_{j-1}, z_{j+1}, \ldots, z_{p+1}\right] \cdot\left[z_{j}, w_{1}, \ldots, w_{p-1}\right]=0 .
$$

However, if the functions $\mathbf{u}^{\alpha}$ are homogeneous polynomials, or, more generally, homogeneous functions of $x$, then there are additional relations stemming from the syzygy between the brackets and the symbolic factors of the first kind

$$
\sum_{j=1}^{p+1}\left[z_{1}, \ldots, z_{j-1}, z_{j+1}, \ldots, z_{p+1}\right] \cdot\left(z_{j}, x\right)=0,
$$

even though the latter factors do not explicitly appear in the transform representation of $P$. We do not have space to go into the full details of the interplay between the invariant differential polynomials and the classical invariants of homogeneous polynomials, but there are three points to note. First, there are far more invariant differential polynomials than classical invariants because we only have "half" the number of syzygies at our disposal in 
the former case. In particular, the invariant differential polynomials are not an algebraically finitely generated ideal. Second, the first fact immediately leads to a number of "universal identities" which are valid for arbitrary homogeneous functions, all of which come from differential polynomials which give the same classical invariant when evaluated on homogeneous polynomial functions. The simplest example is the invariant differential polynomial whose transform is $\left[z_{1} z_{2}\right]^{2}\left[z_{1} z_{3}\right]\left[z_{2} z_{3}\right]$, but whose corresponding umbral representation when $\mathbf{u}$ is a form always vanishes. The differential polynomial has the explicit formula

$$
u_{11}\left(u_{112} u_{222}-u_{122}^{2}\right)-u_{12}\left(u_{111} u_{222}-u_{112} u_{122}\right)+u_{22}\left(u_{111} u_{122}-u_{112}^{2}\right)=0,
$$

which vanishes for all homogeneous functions $u\left(x^{1}, x^{2}\right)$, but is certainly not zero for all smooth functions u. Finally, the classical invariant ${ }^{\text {th }}$ eoretic concept of a perpetuant, [8], [10], which is usually referred to as "an invariant of a form of infinite degree", can be reinterpreted in this light as an invariant differential polynomial of a homogeneous function of non-integral degree of homogeneity, i.e. $u(\lambda x)=\lambda^{\alpha} \cdot u(x)$, where $\alpha$ is not an integer (in particular, $u$ is not a polynomial). This is because all the syzygies of the second kind involve factors like $(\alpha-k), k \in \mathbb{Z}$, so certain relations among bracket polynomials degenerate when $\alpha$ happens to be integral. These remarks will be treated in greater detail in a forthcoming paper.

\section{Characterization of Homogeneous Divergences.}

The solution of problem 1 of the introduction using the transform proceeds as follows. First, for later reference, we determine the transform of a Jacobian determinant

$$
J=\frac{\partial\left(u_{I_{1}}, \ldots, u_{L_{r}}\right)}{\partial\left(x^{k_{1}}, \ldots, x^{k_{r}}\right)}=\operatorname{det}\left[\frac{\partial u_{I_{v}}}{\partial x^{k_{\mu}}}\right]
$$

of derivatives of $u$. A simple calculation shows that

$$
\hat{\mathrm{J}}=\frac{1}{\mathrm{r} !} \operatorname{det}\left(\mathrm{Z}^{\mathrm{K}}\right) \cdot \operatorname{det}\left(\mathrm{Z}^{d}\right)
$$

where $\mathrm{Z}^{\mathrm{K}}$ denotes the $\mathrm{r} \times \mathrm{r}$ minor consisting of columns $\mathrm{k}_{1}, \ldots, \mathrm{k}_{\mathrm{r}}$ of the matrix $\mathrm{Z}$, and $Z^{d}$ denotes the $r \times r$ matrix of monomials $z_{\mu}^{I}$ which occur in the symmetric powers of the matrix $\mathrm{Z}$. 
Theorem. Let $q=1$. Then every differentially homogeneous divergence $Q=$ Div $P$ is a linear combination of Jacobian determinants of derivatives of $u$.

\section{Proof.}

Consider the transform $\hat{Q}$ of $\mathbf{Q}$. Using the formula for the transform of the total derivatives, we see that $Q$ is a divergence if and only if

$$
\hat{Q}(Z)=\sum_{i=1}^{p}\left(z_{1}^{i}+\ldots+z_{r}^{i}\right) \cdot \hat{P}_{i}(Z)
$$

for polynomials $\hat{\mathrm{P}}_{\mathrm{i}}(\mathrm{Z})$. This means that

$$
\hat{Q}(\mathrm{Z})=\hat{Q}\left(\mathrm{z}_{1}, \ldots, \mathrm{z}_{\mathrm{r}}\right)=0 \quad \text { whenever } \quad \mathrm{z}_{1}+\ldots+\mathrm{z}_{\mathrm{r}}=0,
$$

$z_{i}$ denoting the $i^{\text {th }}$ row of $Z$. Moreover, differential homogeneity of $Q$ implies that $\hat{Q}$ is a homogeneous function of degree $k$ of the rows of $Z$, so

$$
\hat{Q}\left(\lambda_{1} z_{1}, \ldots, \lambda_{\mathrm{r}} z_{\mathrm{r}}\right)=\left(\lambda_{1} \cdot \ldots \cdot \lambda_{\mathrm{r}}\right)^{\mathrm{k}} \cdot \hat{Q}\left(\mathrm{z}_{1}, \ldots, \mathrm{z}_{\mathrm{r}}\right) .
$$

Thus we have the stronger condition

$$
\hat{Q}\left(z_{1}, \ldots, z_{r}\right)=0 \quad \text { whenever } \quad z_{1}, \ldots, z_{r} \text { are linearly dependent. }
$$

If $\mathrm{p}<\mathrm{r}$, it is easy to see that there are no nontrivial such polynomials $\hat{\mathrm{Q}}$. Otherwise, let $\Omega^{*}$ denote the determinantal ideal generated by the $r \times r$ minors $Z^{K}$ of the $r \times p$ matrix $Z$. Then the above condition is equivalent to the fact that $\hat{Q}$ vanishes on the ideal $\mathcal{Q}^{*}$. According to a theorem of Northcott, [13], and Mount, [12], $Q^{*}$ is a prime ideal, and so by the Hilbert Nullstellensatz

$$
\hat{Q}(Z)=\sum_{K}\left(\operatorname{det} Z^{K}\right) \cdot \tilde{R}_{K}(Z)
$$

for some polynomials $\tilde{\mathrm{R}}_{\mathrm{K}}(\mathrm{Z})$. Finally, we use the fact that $\hat{\mathrm{Q}}$ is symmetric; applying the symmetrizing map $\sigma$ to the last formula, we can replace $\tilde{R}_{K}(Z)$ by its skewsymmetrization $\hat{R}_{K}$, which is easily seen to be a linear combination of the power determinants $\operatorname{det}\left(Z^{d}\right)$ occurring in the transform of the Jacobian determinants. Therefore 
$\hat{Q}$ is a linear combination of transforms of Jacobian determinants, and hence inverting the transform proves that $Q$ itself is a linear combination of Jacobian determinants, which completes the proof of the theorem.

\section{Hyperjacobians and Transvectants.}

The corresponding problem for homogeneous higher order divergences is approached in the same manner. We begin by transforming the basic condition

$$
\mathrm{Q}=\Sigma \mathrm{D}_{\mathrm{I}} \mathrm{P}_{\mathrm{I}} \text {, }
$$

into the polynomial equation

$$
\hat{Q}(Z)=\sum\left(z_{1}+\ldots+z_{r}\right)^{I} \cdot \hat{P}_{I}(Z),
$$

where $\left(z_{1}+\ldots+z_{r}\right)^{I}$ denotes the $I^{\text {th }}$ power of the sum of the rows of $Z$. Again, differential homogeneity of $Q$ implies that $\hat{Q}$ is an algebraically homogeneous function of the rows of $Z$, so the above condition is equivalent to the condition that $\hat{Q}$ and all its partial derivatives up to order $k-1$, with respect to the variables $z_{j}^{i}$, lie in the determinantal ideal $Q^{*}$. In other words, $\hat{Q}$ lies in the $k^{\text {th }}$ symbolic power of $Q^{*}$.

At this point, we require an important result of Trung, [22], (see also DeConcini, Eisenbud and Procesi, [5]) that for maximal sized minors of a matrix of independent variables $\mathrm{Z}$, the $\mathrm{k}^{\text {th }}$ symbolic power of the corresponding determinantal ideal $\Omega^{*}$ is the same as the ordinary $k^{\text {th }}$ power of $\Omega^{*}$. Therefore, any such $\hat{Q}$ can be written as a sum of powers of determinants:

$$
\hat{Q}(Z)=\sum\left(\operatorname{det} Z^{I_{1}}\right) \cdot \ldots \cdot\left(\operatorname{det} Z^{I_{k}}\right) \cdot \hat{R}^{d}(Z) .
$$

Moreover, applying $\sigma$ as before, we find that $\hat{R}^{d}(Z)$ can be written as a linear combinations either of power determinants, $\operatorname{det}\left(Z^{d}\right)$, if $\mathbf{k}$ is odd, or power permanants, $\operatorname{perm}\left(Z^{d}\right)$, if $\mathbf{k}$ is even. Thus, to complete the characterization of homogeneous higher order divergences, we need to know which differential polynomials transform to

$$
\left(\operatorname{det} Z^{I_{1}}\right) \cdot \ldots \cdot\left(\operatorname{det} Z^{I_{k}}\right) \cdot\left(\operatorname{det} Z^{\ell}\right), \quad \text { if } k \text { is odd, }
$$

or 


$$
\left(\operatorname{det} Z^{I_{1}}\right) \cdot \ldots \cdot\left(\operatorname{det} Z^{I_{k}}\right) \cdot\left(\text { perm } Z^{d}\right), \quad \text { if } k \text { is even. }
$$

The answer is given by the theory of hyperjacobians, [14]. Rather than give the most general definition of a hyperjacobian, which is a generalization of the classical concept of a Jacobian determinant, we present the second degree examples, from which the general definition can easily be guessed.

Example. A first order hyperjacobian is simply a Jacobian determinant, e.g.

$$
\frac{\partial(u, v)}{\partial\left(x^{1}, x^{2}\right)}=u_{1} v_{2}-u_{2} v_{1}
$$

A second order hyperjacobian is obtained from a first order one by a similar determinantal formula:

$$
\frac{\partial^{2}(u, v)}{\partial\left(x^{1}, x^{2}\right) \partial\left(x^{3}, x^{4}\right)}=\frac{\partial\left(u_{3}, v_{4}\right)}{\partial\left(x^{1}, x^{2}\right)}-\frac{\partial\left(u_{4}, v_{3}\right)}{\partial\left(x^{1}, x^{2}\right)}=u_{13} v_{24}-u_{23} v_{14}-u_{14} v_{23}+u_{24} v_{13}
$$

In particular,

$$
\frac{\partial^{2}(u, v)}{\partial\left(x^{1}, x^{2}\right)^{2}}=u_{11} v_{22}-2 u_{12} v_{12}+u_{22} v_{11}
$$

is a multiple of the second transvectant of $u$ and $v$, and

$$
\frac{\partial^{2}(u, u)}{\partial\left(x^{1}, x^{2}\right)^{2}}=2\left(u_{11} u_{22}-u_{12}^{2}\right)
$$

agrees, up to a factor, with the Hessian of $u$. Similarly, third order hyperjacobians are constructed from second order hyperjacobians by the same determinantal procedure; for example

$$
\frac{\partial^{2}(u, v)}{\partial\left(x^{1}, x^{2}\right)^{2}}=\frac{\partial^{2}\left(u_{1}, v_{2}\right)}{\partial\left(x^{1}, x^{2}\right)^{2}}-\frac{\partial^{2}\left(u_{2}, v_{1}\right)}{\partial\left(x^{1}, x^{2}\right)^{2}}=u_{111} v_{222}-3 u_{112} v_{122}+3 u_{122} v_{112}-u_{222} v_{111}
$$

is a multiple of the third transvectant of $u$ and $v$, cf. [9; page 227]. In general, if $u$ and $v$ are homogeneous polynomials, the $k^{\text {th }}$ transvectant $(u, v)^{(\mathbf{k})}$ is just a multiple of the $k^{\text {th }}$ order hyperjacobian $\partial^{\mathbf{k}}(\mathbf{u}, \mathbf{v}) / \partial\left(\mathbf{x}^{1}, \mathbf{x}^{2}\right)^{\mathbf{k}}$.

The general formula for the hyperjacobian 


$$
\frac{\partial^{k}\left(u^{1}, \ldots, u^{r}\right)}{\partial\left(x^{i_{1}}, \ldots, x^{i}\right) \ldots \partial\left(x^{m_{1}}, \ldots, x^{m^{r}}\right)}
$$

is found inductively on $\mathrm{k}$, using a similar determinantal construction. They can also be constructed using the theory of higher dimensional determinants of higher order Jacobian "matrices", cf. [14]. They provide the natural generalization of the notion of transvectant to higher dimensional problems. Furthermore, their transforms are precisely what is required to complete the analysis of higher order divergences, so we have the following result:

Theorem. A differential polynomial $Q$ is a $\mathbf{k}^{\text {th }}$ order divergence if and only if it is a linear combination of $\mathbf{k}^{\text {th }}$ order hyperjacobians of derivatives of $\mathbf{u}$.

In particular, any $\mathbf{k}^{\text {th }}$ order transvectant is a $\mathrm{k}^{\text {th }}$ order divergence, $\mathbf{a}$ fact that does not appear to have previously been noticed in the literature.

\section{Differential Hyperforms.}

Although we now know that any $\mathbf{k}^{\text {th }}$ order hyperjacobian can be written as a $\mathbf{k}^{\text {th }}$ order divergence, the determination of exactly how to do this is a non-trivial task. One solution to this algebraic problem has resulted in the development of a new theory of higher order differential forms, or "hyperforms". The motivation for this is the observation that the divergence identity for the ordinary Jacobian determinant is equivalent to the differential form identity

$$
d u \wedge d v=d(u \cdot d v)
$$

The goal is to develop a theory of differential forms so that the identity

$$
\frac{\partial^{2}(u, v)}{\partial\left(x^{1}, x^{2}\right)^{2}}=D_{1}^{2}\left(-u_{2} v_{2}\right)+D_{1} D_{2}\left(u_{1} v_{2}+u_{2} v_{1}\right)+D_{2}^{2}\left(-u_{1} v_{1}\right)
$$

for the second order hyperjacobian (second transvectant) translates into an identity of the form

$$
d^{2} u * d^{2} v=d^{2}(d u * d v)
$$

for second order hyperforms. Such a thory has been developed over Euclidean space in the unpublished paper [15], and we here summarize its principal ingredients. 
Let $\lambda$ denote a Young diagram, or shape, and $|\lambda|$ the number of boxes in $\lambda$. Given a finite dimensional real vector space $V$, we let $L_{\lambda} V$ denote the corresponding irreducible representation space of the general linear group GL(V); $L_{\lambda}$ is known as the Schur functor (or shape functor). See [1], [11], [21], for the general theory of Schur functors. Pieri's formula is

$$
\mathrm{V} \otimes \mathrm{L}_{\lambda} \mathrm{V}=\bigoplus_{\mu} \mathrm{L}_{\mu} \mathrm{V}
$$

where the direct sum is over all shapes $\mu$ containing $\lambda$ and having precisely one box more than $\lambda$. For notation, we write $\lambda \subset \mu$ when $\lambda$ is contained in $\mu$, and write $\mu l \lambda$ for the skew shape consisting of all boxes of $\mu$ which do not lie in $\lambda$; thus the above direct sum would be over all $\mu \supset \lambda$ with $|\mu| \lambda \mid=1$. This formula implies the existence of functorial maps

$$
\varphi_{\lambda}^{\mu}: \mathrm{V} \otimes \mathrm{L}_{\lambda} \mathrm{V} \rightarrow \mathrm{L}_{\mu} \mathrm{V}
$$

for any such $\lambda, \mu$, which are uniquely defined up to scalar multiple.

Example. Recall first that the Schur space $L_{\lambda} V$ can be identified with the quotient space of the tensor product of the symmetric powers $\odot_{\lambda_{1}} V \otimes \ldots \otimes \odot_{\lambda_{k}} V$ under the two-sided ideal generated by the Young relations

$$
\sum \hat{x}_{\mathrm{i}} \otimes\left(\mathrm{x}_{\mathrm{i}} \odot \mathrm{y}\right)
$$

for $x_{1}, \ldots, x_{p+1} \in V, y \in \odot_{\ell} V$, and where $\hat{x}_{i}=x_{1} \odot \ldots \odot x_{i-1} \odot x_{i+1} \odot \ldots \odot x_{p+1}$. Therefore, each element $\omega$ of $L_{\lambda} V$ can be identified with an equivalence class in $\odot_{\lambda_{1}} V \otimes \ldots \otimes \odot_{\lambda_{k}} V$, and we can use any element of this equivalence class to represent $\omega$. With this in mind we will write elements of $L_{\lambda} V$ as if they were in $\odot_{\lambda_{1}} V \otimes \ldots \otimes \odot_{\lambda_{k}} V$, but with the understanding that we are allowed to replace such a representative by any equivalent element as prescribed by the Young relations.

We can write down the explicit formulas for the Pieri maps $\varphi_{\lambda}^{\mu}$. The easiest approach is to present a few special cases, from which the general formula can be deduced. First, if $\lambda=(k, l), k \geq \ell$, and $\mu=(k+1, l)$, then 


$$
\varphi_{\lambda}^{\mu}(v \otimes(x \otimes y))=(v \odot x) \otimes y+\frac{1}{k-l+2} \sum_{i=1}^{\ell}\left(x \odot y_{i}\right) \otimes\left(v \odot y x_{i}\right) .
$$

If $\mu=(k, \ell+1)$ (so $k>\ell$ ),

$$
\varphi_{\lambda}^{\mu}(v \otimes(x \otimes y))=x \otimes(v \odot y)
$$

while if $\mu=(k, \ell, 1)$, then

$$
\varphi_{\lambda}^{\mu}(v \otimes(x \otimes y))=x \otimes y \otimes v .
$$

Similarly, if $\lambda=(k, l, m), k \geq \ell \geq m$, and $\mu=(k+1, \ell, m)$, then

$$
\begin{aligned}
& \varphi_{\lambda}^{\mu}(v \otimes(x \otimes y \otimes z))=(v \odot x) \otimes y+\frac{1}{k-l+2} \sum_{i=1}^{\ell}\left(x \odot y_{i}\right) \otimes\left(v \odot \hat{y}_{i}\right) \otimes z+ \\
& +\frac{1}{k-m+3} \sum_{j=1}^{m}\left(x \odot z_{j}\right) \otimes y \otimes\left(v \odot \hat{z}_{j}\right)+\frac{1}{k-l+2} \cdot \frac{1}{k-m+3} \sum_{i=1}^{\ell} \sum_{j=1}^{m}\left(x \odot y_{i}\right) \otimes\left(\hat{y}_{i} \odot z_{j}\right) \otimes\left(v \odot \hat{z}_{j}\right) .
\end{aligned}
$$

while if $\mu=(k, \ell+1, m)$, so $k>\ell$, then

$$
\varphi_{\lambda}^{\mu}(v \otimes(x \otimes y \otimes z))=x \otimes(v \odot y) \otimes z+\frac{1}{l-m+2} \sum_{j=1}^{m} x \otimes\left(y \odot z_{j}\right) \otimes\left(v \odot \hat{z}_{j}\right) .
$$

Also, for $\mu=(k, \ell, m+1)$ (so $\ell>m$ ) and $\mu=(k, \ell, m, 1)$ we have

$$
\varphi_{\lambda}^{\mu}(v \otimes(x \otimes y \otimes z))=x \otimes y \otimes(๗ z), \quad \text { and } \quad \varphi_{\lambda}^{\mu}(v \otimes(x \otimes y \otimes z))=x \otimes y \otimes z \otimes v
$$

respectively. The general formula appears in [15].

In any of the above formulas, we could multiply $\varphi_{\lambda}^{\mu}$ by a constant $c_{\lambda}^{\mu}$, without affecting the functoriality. It turns out that one can choose constants $c_{\lambda}^{\mu}$ in such a way that the resulting Pieri products

$$
\mathrm{v} * \omega=c_{\lambda}^{\mu} \cdot \varphi_{\lambda}^{\mu}(\mathrm{v} \otimes \omega), \quad \mathrm{v} \in \mathrm{V}, \quad \omega \in \mathrm{L}_{\lambda} \mathrm{V}, \quad \mathrm{v} * \omega \in \mathrm{L}_{\mu} \mathrm{V},
$$


commute in the sense that $\mathrm{v} * \mathrm{v} * \omega$ is unambiguously defined as an element of $\mathrm{L}_{\mathbf{v}} \mathrm{V}$ whenever $\lambda \subset v$ and $|v| \lambda \mid=2$, in which case the two $*$ products can be computed in two distinct ways, depending on which box is added on to $\lambda$ first. One possible choice of the factors $c_{\lambda}^{\mu}$ is the following: if $\lambda=\left(\lambda_{1}, \ldots, \lambda_{n}\right)$, and $\mu=\left(\lambda_{1}, \ldots, \lambda_{j-1}, \lambda_{j}+1, \lambda_{j+1}, \ldots\right.$, $\left.\lambda_{n}\right)$, then set

$$
c_{\lambda}^{\mu}=\left\{\begin{array}{cl}
\frac{1}{\lambda_{1}+n}, & j=1 \\
\left(\lambda_{1}-\lambda_{j}+j-1\right) \prod_{k=j+1}^{n+1} \frac{1}{\lambda_{j}-\lambda_{k}+k-j}, & 1<j \leq n \\
1, & j=n+1
\end{array},\right.
$$

where $\lambda_{n+1}=0$ by convention. For instance, if $\lambda=(k, l), \mu=(k+1, l)$, then $c_{\lambda}^{\mu}=\frac{1}{(k+2)}$, while if $\mu=(k, \ell+1), c \lambda^{\mu}=\frac{k-l+1}{\ell+1}$. Finally, let $v$ be a fixed nonzero vector in $v$, and define the map $\psi_{\lambda}^{\mu}: L_{\lambda} V \rightarrow L_{\mu} V$ by

$$
\psi_{\lambda}^{\mu}(\omega)=\mathrm{v} * \omega=c_{\lambda}^{\mu} \cdot \varphi_{\lambda}^{\mu}(\mathrm{v} \otimes \omega), \quad \omega \in \mathrm{L}_{\lambda} \mathrm{V} .
$$

Lemma. Let $\lambda \subset \mu \subset v$, and $\lambda \subset \mu^{\prime} \subset v^{\prime}$ be shapes, with $|\mu| \lambda|=1,| \mu \lambda \lambda \mid=1$, and $|v| \mu|=1| v,\left|\mu^{\prime}\right|=1$ (so $|v| \lambda \mid=2$ ). Then

$$
\psi_{\lambda}^{\nu}=\psi_{\mu}^{\nu} \circ \psi_{\lambda}^{\mu}=\psi_{\mu}^{\nu} \circ \psi_{\lambda}^{\mu^{\circ}}
$$

commute and serve to unambiguously define a map $\psi_{\lambda}^{\nu}$ from $L_{\lambda} V$ to $L_{V} V$.

By the lemma, it is easy to see that, given $v \in V$, we can uniquely define a map $\psi_{\lambda}^{\mu}: L_{\lambda} V \rightarrow L_{\mu} V$ for any shape $\mu$ containing $\lambda$; if $|\mu| \lambda \mid=m$, then we define $\psi_{\lambda}^{\mu}$ by $m-$ fold composition of the basic Pieri maps, adding on one box at a time. The lemma assures us that it does not matter in which order the boxes are added on.

Theorem. Let $0 \neq v \in V$, and let $\psi_{\lambda}^{\mu}: L_{\lambda} V \rightarrow L_{\mu} V, \lambda \subset \mu$, be the corresponding maps of Schur spaces. Then the maps $\psi_{\lambda}^{\mu}$ define an exact hypercomplex over $\mathrm{V}$. Here "hypercomplex", which is a generalization of the usual homological algebraic concept of a complex, means that these maps satisfy the two properties of 
a) Commutativity: Whenever $\lambda \subset \mu$ and $\mu \subset v$, then $\psi_{\lambda}^{\nu}=\psi_{\mu}^{\nu} \circ \psi_{\lambda}^{\mu}$.

b) Closure: Whenever $\lambda \subset v$ and $v 1 \lambda$ contains two or more boxes in any one column, then $\psi_{\lambda}^{\nu}=0$.

Furthermore, the Schur hypercomplex is exact, meaning that

c) Exactness: Suppose $\lambda \subset \mu$ and $\mu \subset v$, and $\mu \mid \lambda$ and $v \mu$ each consist of only one row of boxes, and $v \backslash \lambda$, which consists of two rows of boxes, has two boxes in one and only one column. Then, $\psi_{\mu}^{\nu}(\omega)=0$ for $\omega \in \mathrm{L}_{\mu} V$ if and only if $\omega=\psi_{\lambda}^{\mu}(\theta)$ for some $\theta \in \mathrm{L}_{\lambda} \mathrm{V}$.

(There is a more general statement of exactness which characterizes the kernel of $\psi_{\mu}^{\nu}$ for any $\mu \subset v$, but this is a little harder to state, and the above notion of exactness is sufficient for this more general version to hold; see [15] for details.)

Contained in the Schur hypercomplex, corresponding to the shapes with only one column of boxes, is the standard exterior complex $\omega \rightarrow v \wedge \omega, \omega \in \wedge_{\mathrm{k}} \mathrm{V}$, and in this case, closure and exactness reduce to their more familiar counterparts.

What we are really interested in is the differential counterpart of the Schur hypercomplex. At present, the construction has only been carried out for open subsets $\mathbf{M}$ of Euclidean space $\mathbb{R}^{p}$, but extensions to arbitrary smooth manifolds are possible, once certain technical details regarding changes of variable have been overcome. Let $T^{*} \mathrm{M}$ be the cotangent bundle of $M$, with $\left.T^{*} M\right|_{x}$, which will play the role of our vector space $V$, denoting the cotangent space of $M$ at $x \in M$. Let $\left.\Xi_{\lambda}\right|_{x}=\left.L_{\lambda} T * M\right|_{x}$ be the corresponding Schur space at $x$, with $\Xi_{\lambda}$ the corresponding "Schur bundle", constructed in the exact same manner as the exterior powers $\Lambda_{\mathbf{k}} \mathrm{T}^{*} \mathrm{M}$, which are just special cases when $\lambda$ has only one column of boxes. A differential hyperform of shape $\lambda$ will be a section of $\boldsymbol{\Xi}_{\lambda}$. More explicitly, let $\mathrm{dx}^{1}, \ldots, \mathrm{dx} \mathrm{p}^{\mathrm{p}}$ be the standard basis of $\mathrm{T}^{*} \mathrm{M}$. Then corresponding to every Young tableau of shape $\lambda$ whose entries are chosen from the set of indices $\{1, \ldots, p\}$, there is an element $\mathrm{dx}_{\mathrm{T}}$ of $\Xi_{\lambda}$. The elements $\mathrm{dx}_{\mathrm{T}}$ corresponding to standard tableaux form a basis of $\Xi_{\lambda}$, cf. [1]. (Here standard means that the rows of the tableau are nondecreasing and the columns strictly increasing.) Therefore any differential hyperform can be written in the form 


$$
\omega=\sum \mathrm{f}_{\mathrm{T}}(\mathrm{x}) \cdot \mathrm{dx}_{\mathrm{T}}
$$

where the sum is over all standard tableaux of shape $\lambda$. If $\lambda \subset \mu$ and $\mu$ differs from $\lambda$ by only one box, then we define the differential of $\omega$ to be the differential hyperform

$$
\mathrm{d} \omega=\mathrm{d}_{\lambda}^{\mu} \omega=\sum_{\mathrm{T}} \mathrm{df}_{\mathrm{T}}(\mathrm{x}) * \mathrm{dx}_{\mathrm{T}}=\sum_{\mathrm{T}} \mathrm{c}_{\lambda}^{\mu} \cdot \varphi_{\lambda}^{\mu}\left(\mathrm{df}_{\mathrm{T}}(\mathbf{x}) \otimes \mathrm{dx}_{\mathrm{T}}\right),
$$

where $d f_{T} \in T * M$ denotes the ordinary differential of the coefficient function $f_{T}$. The algebraic commutativity lemma immediately implies the commutativity of the differentials: Let $\lambda \subset \mu \subset v$, and $\lambda \subset \mu^{\prime} \subset v$ be shapes, with $|\mu| \lambda|=1,| \mu^{\prime}|\lambda|=1$, and $|v| \mu \mid=1$, $|v| \mu^{\prime} \mid=1$ (so $|v| \lambda \mid=2$ ). Then $d_{\mu^{v}}^{v} \circ d_{\lambda}^{\mu}=d_{\mu^{\prime}}^{v} \circ d_{\lambda}^{\mu^{\prime}}$ commute and serve to unambiguously define a second order differential $d_{\lambda}^{v}$ from $\Xi_{\lambda}$ to $\Xi_{v}$. Therefore, by composition, we can define a differential $d=d_{\lambda}^{\mu}: \Xi_{\lambda} \rightarrow \Xi_{\mu}$ whenever $\lambda \subset \mu$. The main result from [15] is:

Theorem. Let $M \subset \mathbb{R}^{p}$ be a star-shaped domain. Then the differential hypercomplex defined by the differentials $\mathrm{d}_{\lambda}^{\mu}: \Xi_{\lambda} \rightarrow \Xi_{\mu}$ on hyperforms is an exact hypercomplex.

Contained within the differential hypercomplex, corresponding to those shapes with only one column, is the ordinary deRham complex over $\mathrm{M}$, where closure and exactness reduce to their usual meanings. There are also a host of other interesting subcomplexes of the full hypercomplex, but space precludes us presenting this in any detail here. If $M$ is not star-shaped, then one can use the failure of the differential hypercomplex to be exact to define new "hypercohomology" over $M$, but this has not been investigated at all.

If $\lambda=0$ is the "empty shape", then $\Xi_{\lambda \mid x}=\mathbb{R}$, and a "0-hyperform" is just an ordinary real-valued function. If $\mu=(k)$ consists of a single row, so $\Xi_{\mu}=\odot_{k} T^{*} M$ is the $\mathrm{k}^{\text {th }}$ symmetric power of $T^{*} \mathrm{M}$, then $d_{0}^{\mu} \mathrm{u}=\mathrm{d}^{\mathrm{k}} \mathrm{u}$ is just the $\mathrm{k}^{\text {th }}$ differential of the function u, i.e.

$$
d^{k} u=\sum_{I}\left(\begin{array}{l}
k \\
I
\end{array}\right) u_{I} \cdot d x_{I}
$$

the sum being over all multi-indices $I$ of order $k$, with $u_{I}$ denoting the corresponding $\mathrm{k}^{\text {th }}$ order partial derivative. One immediate consequence of the exactness of the differential hypercomplex is that, provided $M$ is star-shaped, a section $\omega$ of $\Xi_{\mu}=\odot_{k} T^{*} M$ is a $k^{\text {th }}$ 
order differential, $\omega=d^{k}$ for some function $f$, if and only if $d_{\mu}^{v} \omega=0$, where $v=(k, 1)$ is the two-rowed shape with one box in the second row.

Turning to the hyperjacobian identities, let $\mu$ be the rectangular shape consisting of $p$ rows, each consisting of $k$ boxes, i.e. $\mu=(k, k, \ldots, k)$. It can be shown, using the Littlewood-Richardson rule, that the representation space $L_{\mu} V$ occurs with multiplicity one in the $\mathrm{p}$-fold tensor product $\otimes_{\mathrm{p}} \odot_{\mathrm{k}} \mathrm{V}$ of the $\mathrm{k}^{\text {th }}$ symmetric power of $\mathrm{V}$. Hence there is, up to constant multiple, just one functorial map $\pi: \otimes_{\mathrm{p}} \odot_{\mathrm{k}} \mathrm{V} \rightarrow \mathrm{L}_{\mu} \mathrm{V}$, and that is given by identifying $d^{k} u^{1} \otimes \ldots \otimes d^{k} u^{p} \in \otimes_{p} \odot_{k} V$ as an element of $L_{\mu} V$. A straight-forward calculation using the straightening rule shows that the coefficient of $\left(\mathrm{dx}^{1}\right)^{\mathbf{k}} \otimes \ldots \otimes\left(\mathrm{dx}^{\mathrm{P}}\right)^{\mathrm{k}}$ is the $\mathrm{k}^{\text {th }}$ order hyperjacobian $\partial^{\mathrm{k}}\left(\mathrm{u}^{1}, \ldots, \mathrm{u}^{\mathrm{p}}\right) / \partial\left(\mathrm{x}^{1}, \ldots, \mathrm{x}^{\mathrm{p}}\right)^{\mathrm{k}}$. (This can be generalized to other hyperjacobians by changing the number of $u^{\prime}$ s.) For example, as an element of $\Xi_{\mu}$, where $\mu=(2,2)$,

$d^{2} u \otimes d^{2} v$

$=\left\{u_{11}\left(d x^{1}\right)^{2}+2 u_{12} d x^{1} \odot d x^{2}+u_{22}\left(d x^{2}\right)^{2}\right\} \otimes\left\{u_{11}\left(d x^{1}\right)^{2}+2 u_{12} d x^{1} \odot d x^{2}+u_{22}\left(d x^{2}\right)^{2}\right\}$

$$
=\left\{u_{11} v_{22}-2 u_{12} v_{12}+u_{22} v_{11}\right\}\left(d x^{1}\right)^{2} \otimes\left(d x^{2}\right)^{2}
$$

since

$$
\left(d x^{1}\right)^{2} \otimes\left(d x^{2}\right)^{2}+2\left(d x^{1} \odot d x^{2}\right) \otimes\left(d x^{1} \odot d x^{2}\right)=0
$$

by the Young relation.

Furthermore, let $\lambda$ consist of $p-1$ rows with $k$ boxes in each row. Let $\mathrm{k}=\mathrm{sp}+\mathrm{t}$, where $0 \leq \mathrm{t}<\mathrm{p}$. Then $\mathrm{L}_{\lambda} \mathrm{V}$ occurs with multiplicity one in the tensor product $\otimes_{\mathrm{t}} \Theta_{\mathrm{k}-\mathrm{s}-1} \mathrm{~V} \otimes \otimes_{\mathrm{p}-\mathrm{t}} \Theta_{\mathrm{k}-\mathrm{s}} \mathrm{V}$, and so, up to constant multiple, there is a unique functorial map $\hat{\pi}: \otimes_{\mathrm{t}} \odot_{\mathrm{k}-\mathrm{s}-1} \mathrm{~V} \otimes \otimes_{\mathrm{p}-\mathrm{t}} \odot_{\mathrm{k}-\mathrm{s}} \mathrm{V} \rightarrow \mathrm{L}_{\lambda} \mathrm{V}$. (This is a little harder to describe explicitly; see [15].) Fixing this multiple appropriately, we find that the hyperform identity

$$
\begin{aligned}
& d^{k} u^{1} \otimes \ldots \otimes d^{k} u^{p}= \\
& \quad d^{k}\left(d^{k-s-1} u^{1} \otimes \ldots \otimes d^{k-s-1} u^{t} \otimes d^{k-s} u^{t+1} \otimes \ldots \otimes d^{k-s} u^{p}\right)
\end{aligned}
$$


is equivalent to the expression of the above $\mathrm{k}^{\text {th }}$ order hyperjacobian as a $\mathrm{k}^{\text {th }}$ order divergence. Here we are identifying the hyperform in parenthesis with its image in $\Xi_{\lambda}$ under the map $\hat{\pi}$, and $d^{k}=d_{\lambda}^{\mu}$.

Example. For the Hessian, we need to look at the identity

$$
d^{2} u \otimes d^{2} v=d^{2}(d u \otimes d v),
$$

where $d^{2} u \otimes d^{2} v \in \Xi_{[2,2]}$, and $d u \otimes d v \in \Xi_{[2]}$. We find

$$
d u \otimes d v=-\frac{1}{2} d u \odot d v=-\frac{1}{2}\left\{u_{1} v_{1}\left(d x^{1}\right)^{2}+\left(u_{2} v_{1}+u_{1} v_{2}\right) d x^{1} \odot d x^{2}+u_{2} v_{2}\left(d x^{2}\right)^{2}\right\}
$$

and

$$
\begin{aligned}
d^{2}(d u \otimes d v) & =-\frac{1}{2}\left\{d^{2}\left(u_{1} v_{1}\right) *\left(d x^{1}\right)^{2}+d^{2}\left(u_{2} v_{1}+u_{1} v_{2}\right) * d x^{1} \odot d x^{2}+d^{2}\left(u_{2} v_{2}\right) *\left(d x^{2}\right)^{2}\right\} \\
& =\left\{-D_{2}^{2}\left(u_{1} v_{1}\right)+D_{1} D_{2}\left(u_{2} v_{1}+u_{1} v_{2}\right)-D_{1}^{2}\left(u_{2} v_{2}\right)\right\} \cdot\left(d x^{1}\right)^{2} \otimes\left(d x^{2}\right)^{2}
\end{aligned}
$$

and we recover the second order transvectant identity. See [14], [15], for further identities of this type.

\section{References}

[1] Akin, K., Buchsbaum, D. A. and Weyman, J., Schur functors and Schur complexes, Adv. in Math. 44 (1982), 207-278.

[2] Ball, J. M., Currie, J.C. and Olver, P.J., Null Lagrangians, weak continuity, and variational problems of arbitrary order, J. Func. Anal. 41 (1981), 135174.

[3] Coleman, B.D. and Noll, W., The thermodynamics of elastic materials with heat conduction and viscosity, Arch. Rat. Mech. Anal. 13 (1963), 167-178.

[4] Cushman, R. and Sanders, J.A., Nilpotent normal forms and representation theory of sl(2,R), Univ. of Amsterdam, Report \#301, 1985.

[5] DeConcini, C., Eisenbud, D. and Procesi, C., Young diagrams and determinantal varieties, Invent. Math. 56 (1980), 129-165. 
[6] Dunn, J.E. and Serrin, J., On the thermomechanics of interstitial working, Arch. Rat. Mech. Anal. 88 (1985), 95-133.

[7] Gel'fand, I.M. and Dikii, L.A., Asymptotic behaviour of the resolvent of SturmLiouville equations and the algebra of the Korteweg-deVries equations, Russ. Math. Surveys 30 (1975), 77-113.

[8] Grace, J.H. and Young, A., The Algebra of Invariants, Cambridge Univ. Press, Cambridge, 1903.

[9] Gurevich, G.B., Foundations of the Theory of Algebraic Invariants, P. Noordhoff Ltd., Groningen, Holland, 1964.

[10] Kung, J.P.S. and Rota, G.-C., The invariant theory of binary forms, Bull. Amer. Math. Soc. 10 (1984), 27-85.

[11] Lascoux, A. Syzygies des varietés determinantales, Adv. in Math. 30 (1978), 202237.

[12] Mount, K.R., A remark on determinantal loci, J. London Math. Soc. 42 (1967), 595-598.

[13] Northcott, D.G., Some remarks on the theory of ideals defined by matrices, Quart. J. Math. Oxford 85 (1963), 193-204.

[14] Olver, P.J., Hyperjacobians, determinantal ideals and weak solutions to variational problems, Proc. Roy. Soc. Edinburgh 95A (1983), 317-340.

[15] Olver, P.J., Differential hyperforms I, Univ. of Minn. Math. Report 82-101, 1983.

[16] Olver, P.J., Conservation laws and null divergences, Math. Proc. Camb. Phil. Soc. 94 (1983), 529-540.

[17] Olver, P.J., Conservation laws and null divergences II. Nonnegative divergences, Math. Proc. Camb. Phil. Soc. 97 (1985), 511-514.

[18] Olver, P.J., Applications of Lie Groups to Differential Equations, Graduate Texts in Mathematics, vol. 107, Springer-Verlag, New York, 1986.

[19] Shakiban, C., A resolution of the Euler operator II, Math. Proc. Camb Phil. Soc. 89 (1981), 501-510.

[20] Shakiban, C. An invariant theoretic characterization of conservation laws, Amer. J. Math. 104 (1982), 1127-1152.

[21] Towber, J., Two new functors from modules to algebras, J. Algebra 47 (1977), 80-104.

[22] Trung, N.V., On the symbolic powers of determinantal ideals, J. Algebra 58 (1979), 361-379. 